\title{
THE INFLUENCE OF LIQUID LOAD MOTION ON ROLLOVER STABILITY OF ROAD TANKERS
}

Rollover threshold is an essential factor in research into road tanker behavior since the accidents involving these vehicles frequently have fatal consequences, especially when their load is of a flammable or toxic nature.

The following article presents a method of identifying the influence of liquid motion in a partially filled road tanker. The method is based on simulation using a mathematical model developed with the help of MSA (Multi-body System Analysis) software. This software enables the user to evaluate all the factors that influence rollover stability. Mechanical pendulum methodology has been used to simulate the liquid motion.

The road tanker model was tested in compliance with the results obtained by measuring carried out on a SCANIA 124 L truck combined with a ZVVZ trailer. Simulation tests were carried out for the drive on a circular track and in a drive around a roundabout, which is considered the most dangerous manoeuvre in respect to vehicle rollover.

\section{Introduction}

Vehicle rollover has always resulted in a serious accident, especially for heavy trucks. Rapid development of road transport combined with significant growth in the number of road accidents has been recently recorded with heavy trucks representing a major share. Most accidents are caused by heavy road vehicle rollover. Heavy vehicle rollover appears to be a serious problem requiring careful evaluation especially for the vehicles transporting hazardous loads (i.e. road tankers), which can be classified as a subject of ADR regulations.

There are roughly 1,500 types of material transported on the roads that are classified as dangerous. This number is far from being finite, as the list of dangerous substances has been continuously growing. It is estimated that in the year 2010, dangerous substances will make $80 \%$ of all the transported goods.

Regarding this aspect, heavy road truck rollover, especially road tankers, is clearly a serious and topical problem. This fact is supported by the fact that rollover accidents have recently been the focal point of numerous research works.

\section{Problems of Vehicle Rollover}

Heavy vehicle stability in cornering is primarily influenced by the following four factors: centre of gravity, wheel gauge, road turn diameter and speed of the vehicle. Besides these factors, there is also secondary rollover effect - suspended mass roll (vehicle body), which depends on suspension characteristics. Centrifugal force in cornering develops vehicle roll around its rollover centre line. This roll will generate the rollover of a vehicle equipped with soft suspension at lower speeds than that of a stiff suspension. Shift and lift of the center of gravity will cause an increase in the roll moment that adds to further reduction of the roll speed. This applies even in case that the load slips on the bearing length to the side. All the above-indicated effects can be represented by static forces, and these can be simulated on a tilting table.

The real situation is more complicated due to a variety of different dynamic effects; therefore a similar simple illustration is not sufficient. It is especially so in the case of road tankers that are difficult to describe because of the effects of free liquid motion in the tank.

\section{Liquid - Vehicle Interaction}

Numerous studies have been carried out examining the interaction of liquid sloshing and vehicle rollover. Most of the studies done in the 80s and 90s of the previous century are based on computer simulations using models with differing capabilities of processing. The most significant problem is the method utilized to simulate liquids in free motion. The most suitable seems to be the method published by Ranganathan et al.[1] in 1993. This method is based on substituting the liquid motion by a pendulum, whose parameters are derived from the amount of liquid in the tank. The structure, including the principal parameters of the pendulum, is given in Figure 1.

Description of the items in Figure 1:

A pendulum centre

$a_{A}, a_{k}, a_{0}$ lateral acceleration

$M \quad$ weight of moving liquid

$m_{0} \quad$ weight of steady state liquid

$l_{k} \quad$ pendulum length depending on the level of tank filling

\footnotetext{
* Miroslav Tesař

University of Pardubice, The Jan Perner Transport Faculty, Czech Republic, E-mail: Miroslav.Tesar@upce.cz
} 


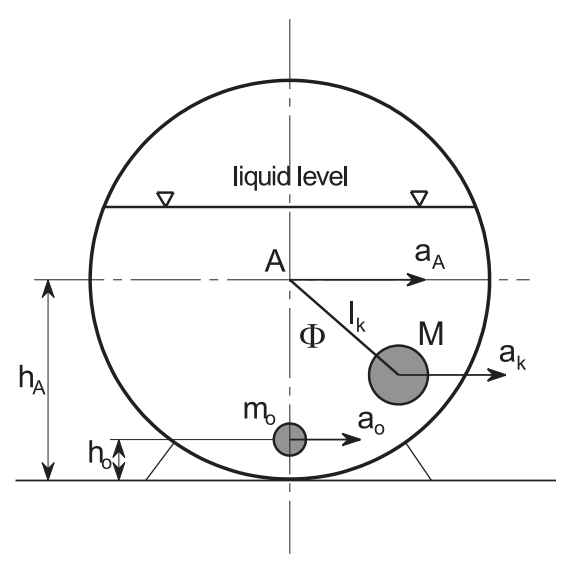

Fig. 1: Liquid motion demonstrated by means of mathematic pendulum

The method for calculation of motion of pendulum is the same as the method applied for mathematic model of vehicle.

This method makes use of markers. In accordance with ISO 2382 a marker is symbol for indication of location.

Every primary element is localized with a marker having a local coordinate system whose location in the global coordinate system $a$ is defined by the origin location and orientation in the global coordinate system by means of transformation matrix $T_{a b}$

Transformation matrix $T_{a b}$ of motion $b: a$ is

$$
\begin{aligned}
& T_{a b}=T_{1 n}=\left[\begin{array}{cccc}
a_{11} & a_{12} & a_{13} & a_{1} \\
a_{21} & a_{22} & a_{23} & a_{22} \\
a_{31} & a_{32} & a_{33} & a_{3} \\
0 & 0 & 0 & 0
\end{array}\right]=\left[\begin{array}{cc}
S_{a b} & u_{a b} \\
0 & 1
\end{array}\right] \\
& S_{a b}=\left[a_{i k}\right] \\
& u_{a b}=\left\lceil a_{1}, a_{2}, a_{3}\right\rceil
\end{aligned}
$$

where: $S_{a b}$ - is matrix of heading angle cosines

$$
u_{a b} \text { - is column vector of origin } O_{n} \text { in space } a \text {. }
$$

Resulting transformation matrix $T_{a b}$ or $T_{1 n}$ of finite location of the local coordinate system $b$ is defined by a product of transformation matrices of basic motions according to:

$$
T_{1 n}=T_{12} \cdot T_{23} \cdot T_{34} \cdot T_{n-1, n}
$$

For example, for fluid sloshing (pendulum motion) the point $M$ location (Fig. 1.) is fixed with marker $b$ defined by matrix equation (5). This equation describes the motion of point $M$ of local coordinate system $n$ (marker $b$.) in space 1 (tank, space $a$ ). This equation is matrix parametric equation of point:

$$
r_{1 M}=T_{12} T_{23} T_{34} T_{n+1, n} \cdot r_{n M}=T_{1 n} \cdot r_{n M}
$$

where: $r_{1 M}$ - is column vector of point $\mathrm{M}$ in coordinate system 1 (equation 6)

$$
\begin{gathered}
\text { (equation 7) } \\
r_{1 M}=\left\lceil x_{1 M}, y_{1 M}, z_{1 M}, 1\right\rceil \\
r_{n M}=\left\lceil x_{n M}, y_{n M}, z_{n M}, 1\right\rceil
\end{gathered}
$$$$
r_{n M} \text { - is column vector of point } \mathrm{M} \text { in coordinate system } n
$$

Vectors of speed $v_{1 M}$ and vectors of acceleration $a_{1 M}$ in coordinate system 1 (global coordinate system $a$ ) are the following:

$$
\begin{aligned}
& v_{1 M}=\dot{T}_{1 n} \cdot r_{n M} \\
& a_{1 M}=\ddot{T}_{1 n} \cdot r_{n M}
\end{aligned}
$$

We have been involved in rollover stability for several years. The method presented above was used for evaluating the influence of liquid sloshing on road tanks stability. A pendulum substituting free liquid motion was integrated in the mathematical model of a road tank.

The overall layout of the model is demonstrated in Figure 2.

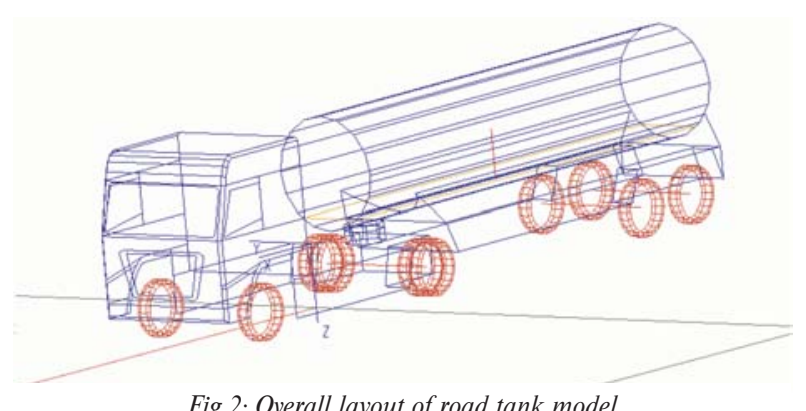

Fig.2: Overall layout of road tank model

The mathematical model of a road tanker was made with the help of MSA (Multi-body System Analysis) software. This software was developed for static, kinematic and dynamic analysis of complex mathematic systems. Other software modules enable users to model even such complex dynamic processes as a road vehicle run. The software contains a group of user variable modules: DRIVE/Transmission to select the vehicle transmission parameters, DRIVE/Driver to define the nominated road route combined with various driver characteristics and DRIVE/Tire - a tire defining module.

The mathematical model is assembled in a way that includes all the factors affecting rollover stability. Weights and inertia momentums of a truck and trailer suspension, inertia momentums of un-sprung mass reduced to wheels, weight and inertia momentum of free liquid in a road tank are the factors integrated in the mathematic model. The model structure enables one to define any suspension type involving the various characteristics of springing elements and shock absorbers including flexible backstops.

To verify the mathematical model, the following values were measured on a real vehicle on a circular track: speed, lateral accel- 
eration, suspended mass rollover. The same values were computed using the mathematical simulation of the identical vehicle and drive. Comparison of the results is given in the charts in Figure 3. The results show a very high level of coincidence proving that a mathematic model can be used for testing the influence of liquid on rollover stability.
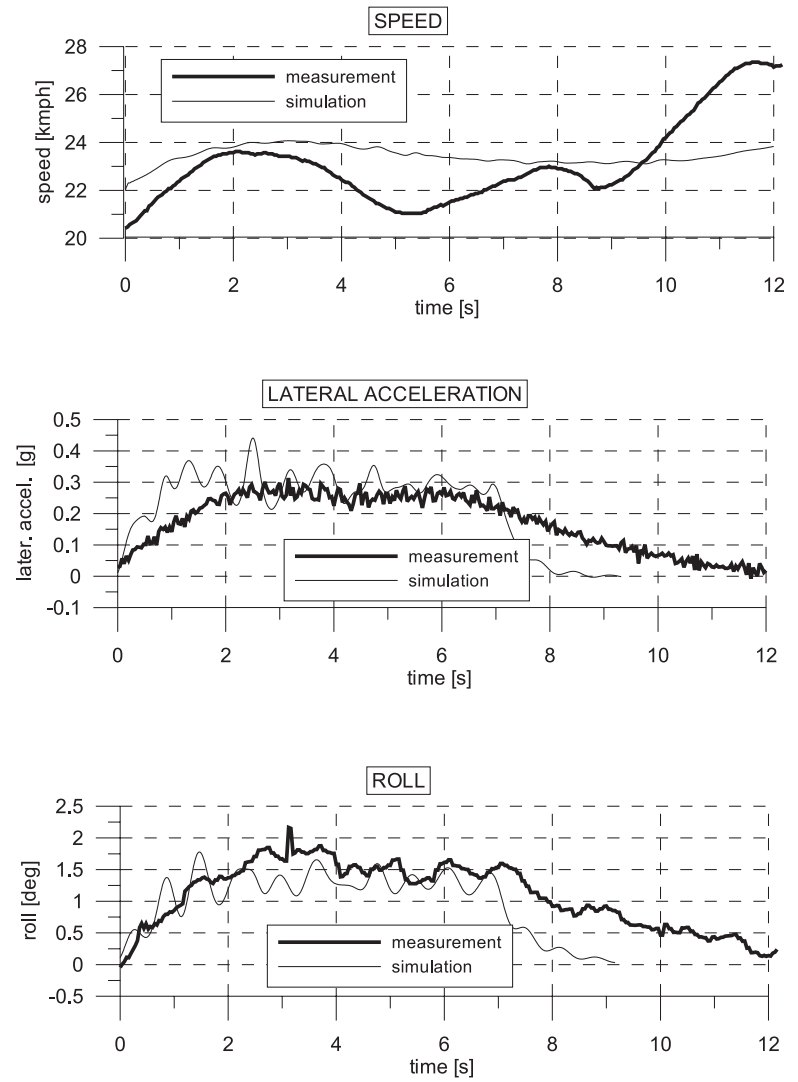

Fig. 3: Results of mathematic model verification

Numerous simulation tests were carried out with this model to verify the influence of liquid sloshing on rollover stability. The individual simulation tests were carried out with different levels of tank filling. Driving on a circular track and drive through a roundabout were selected for testing.

\section{Results of Simulation Tests}

The tests results showed that liquid sloshing depends on two major factors: firstly, on the level of tank filling and secondly, on the way in which the vehicle is actually driven. This assumption is based on the comparison of simulation tests results in individual ways of driving.

The drive on the circular track can be considered a steady state with its results approaching the static state. Comparing lateral acceleration and roll values in driving on circular track at constant speed proved this. The results are given in Figures 4 and 5.

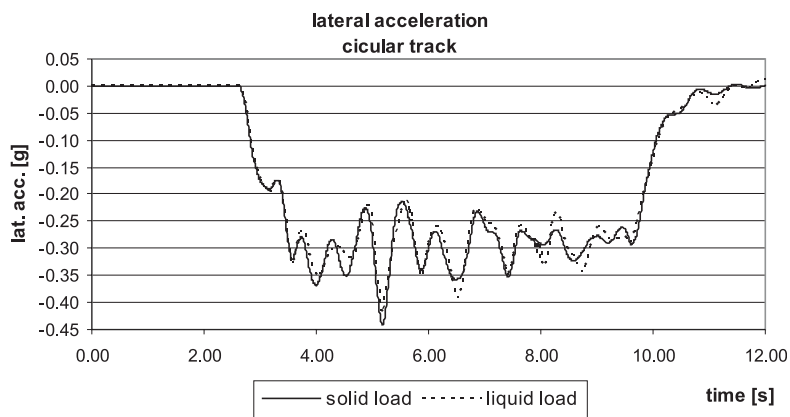

Fig. 4: Comparing lateral acceleration with solid and liquid load

roll

circular track

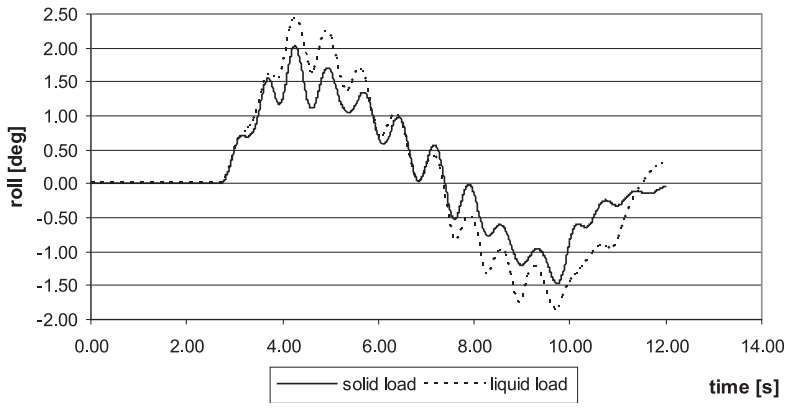

Fig. 5: Comparing the roll with solid and liquid load

The results show that the value of lateral acceleration stays almost unchanged. The roll angle is greater with a liquid load. This is caused by the shift in the center of gravity of liquid sloshing to the sides and upwards. The ratio between the roll and stabilizing moment changes and the vehicle rolls more extensively. Yet is this change not large enough to cause the vehicle rollover.

A different situation occurs when the vehicle drives through a roundabout. Roundabouts are considered the most dangerous manoeuvre from the rollover point of view. Figures 6 and 7 show the simulation of a drive through a roundabout.

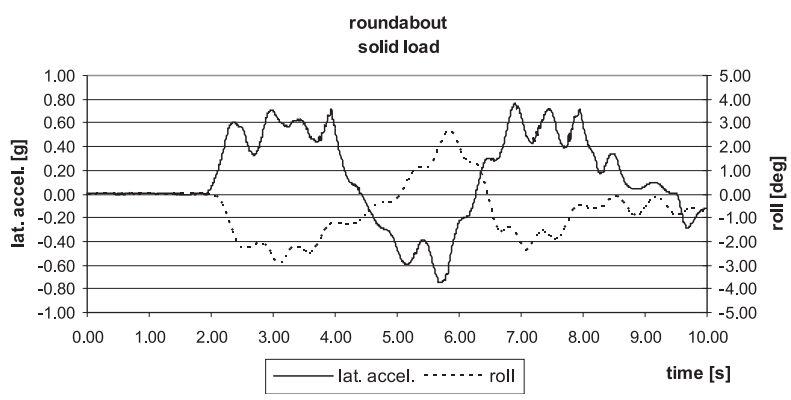

Fig. 6: Roll and acceleration course with solid load.

The record of a drive through a roundabout with solid load (Fig. 6) shows that the course of lateral acceleration and vehicle roll correspond to the changes in entering and exiting the roundabout. The vehicle negotiated the whole track without any problems. 


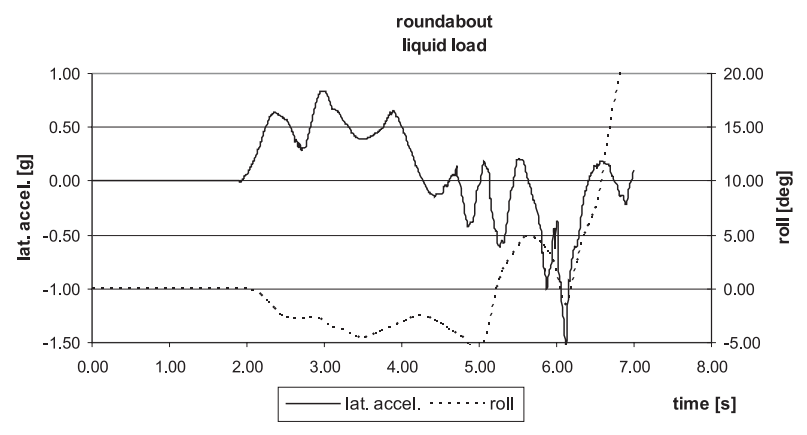

Fig. 7: Roll and acceleration course with liquid load

Figure 7 shows the influence of liquid on the vehicle behaviour in driving through a roundabout. When entering the roundabout, the vehicle drives through the right turn, then through a roundabout with a constant diameter. The change of direction is demonstrated in both the charts at the time of around the 5th second. The vehicle starts to roll in the opposite direction. Then it exits the roundabout, which leads to a rapid change of direction. Another roll appeared between the 6th and 7th second at the vehicle with solid load. The vehicle with liquid load also starts to roll but the back motion of sloshing liquid causes a sudden transfer of the gravity center and the vehicle starts to roll over. Even an experienced driver would not be probably able to prevent the rollover at this stage. At the $7^{\text {th }}$ second the vehicle rolls over. This is the time when the vehicle with solid load drives through the right turn in exiting the roundabout. The vehicle behavior can be seen in the animation of the simulation outputs. Figure 8 shows the vehicle with solid load and Figure 9 shows the vehicle with liquid load at the same instant of time in driving through the roundabout. The liquid moves and the trailer wheels on one side lift. In the back motion the rolling energy reaches the values that cause the vehicle rollover.

\section{Conclusion}

The results of simulation tests demonstrate that this method can be used to assess the influence of liquid sloshing on rollover

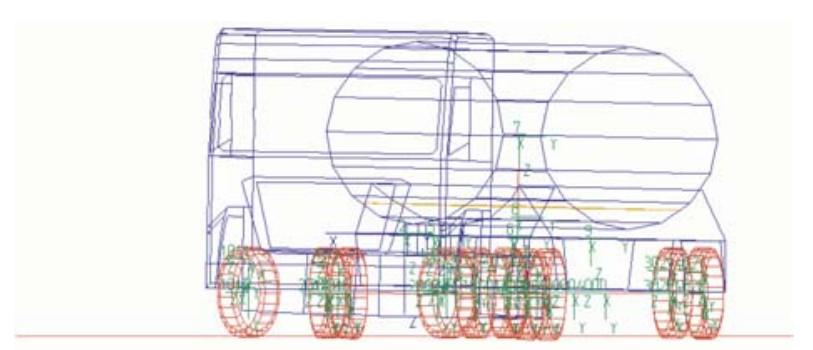

Fig. 8: Vehicle with solid load

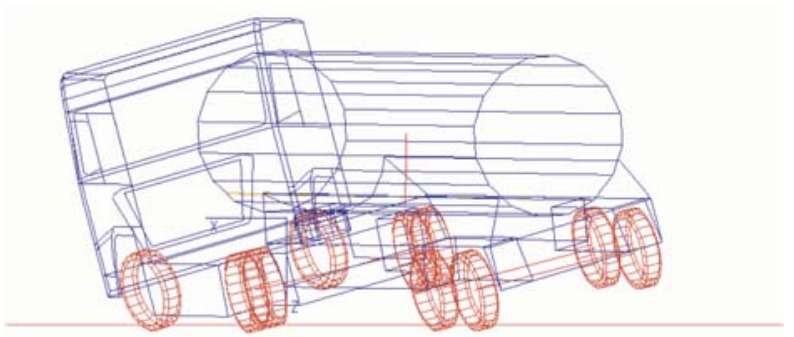

Fig. 9: Vehicle with liquid load. At the same instant of time, the vehicle with liquid load rolls over due to the effect of liquid sloshing.

stability of road tanks. Significant factor is the level of tank filling. Another aspect to consider is the way of driving. It seems that a drive through a roundabout followed by a rapid change of direction towards the exit is the most dangerous manoeuvre. Numerous simulation tests will have to be carried out to find out the relations between tank filling, speed and the type of manoeuvre. The results will contribute to a deeper insight into complex dynamic processes that significantly influence rollover stability of partially filled road tanks. This will help to reduce the probability of road accidents of vehicles transporting dangerous load whose results are in most cases fatal.

\section{References}

[1] RANGAnAthan, R., YING, Y., MILES, J. B.: Development of Mechanical Analogy Model to Predict the Dynamic Behavior of Liquids in Partially Filled Tank Vehicles. SAE Paper 942307.

[2] TESAŘ, M., ET ALL.: Research of evaluation of heavy vehicles rolls stability methods. Final report. Project MoT. University of Pardubice, The Jan Perner Transport Faculty, 2004. 